\title{
Asymmetric nuclear matter: the role of the isovector scalar channel
}

\author{
B. Liu ${ }^{1,2}$, V. Greco ${ }^{1}$, V. Baran ${ }^{1,3}$, M. Colonna ${ }^{1}$ and M. Di Toro ${ }^{1}$ \\ ${ }^{1}$ Laboratorio Nazionale del Sud, Via S. Sofia 44, I-95123 Catania, Italy \\ and University of Catania \\ ${ }^{2}$ Institute of High Energy Physics, Chinese Academy of Sciences, Beijing 100039, China \\ ${ }^{3}$ NIPNE-HH, Bucharest, Romania
}

We try to single out some qualitative new effects of the coupling to the $\delta$-isovector-scalar meson introduced in a minimal way in a phenomenological hadronic field theory. Results for the equation of state $(E O S)$ and the phase diagram of asymmetric nuclear matter $(A N M)$ are discussed. We stress the consistency of the $\delta$-coupling introduction in a relativistic approach.

New contributions to the slope and curvature of the symmetry energy and the neutron-proton effective mass splitting appear particularly interesting.

A more repulsive EOS for neutron matter at high baryon densities is expected.

Effects on new critical properties of warm $A N M$, mixing of mechanical and chemical instabilities and isospin distillation, are also presented. The $\delta$ influence is mostly on the isovectorlike collective response.

The results are largely analytical and this makes the physical meaning quite transparent. Implications for nuclear structure properties of drip-line nuclei and for reaction dynamics with Radioactive Beams are finally pointed out.

PACS numbers: 21.65.+f, 21.30.Fe, 25.70.Pq, 24.10.Jv

\section{INTRODUCTION}

Hadronic effective field theories [quantum hadrodynamics, $Q H D]$ represent a significant improvement in the understanding of static and dynamical properties of nuclear matter and finite nuclei, described as strongly interacting systems of baryons and mesons. The main application of this approach has been the Relativistic Mean Field theory $(R M F)$ [1]2], extremely successful in nuclear structure studies $[3$ - 5 .

In recent years, due to the new possibilities opened by the Radioactive Beam facilities, the interest has moved towards a microscopic description of asymmetric nuclear systems, from the stability of drip-line nuclei to the phase diagram of asymmetric nuclear matter. The connection to astrophysics problems, supernovae explosions and neutron stars, is also quite evident [6] 9 . The isovector channel has been introduced through a coupling to the charged vector $\rho$-meson. In the Hartree approximation of the $R M F$ approach it leads to a simple linear increase of the symmetry term with the baryon density, without neutron/proton effective mass splitting [10]. A complete study of the corresponding phase diagram for asymmetric nuclear matter has been performed in ref. [11], with a thorough discussion of the new qualitative features of the liquid-gas phase transition.

In this work we focus our attention on the introduction of the coupling to the isovector scalar channel, through the exchange of a virtual charged $\delta\left[a_{0}(980)\right]$ meson. In this way, as well know in the isoscalar channel, we recover the genuine structure of the relativistic interactions, where one has the balance between scalar (attractive) and vector (repulsive) "potentials". Actually the $\delta$-meson exchange is an essential ingredient of all $N N$ realistic potentials and its inclusion in the $Q H D$ scheme has been already suggested [12], also on the basis of a relativistic Brueckner theory 13 15]. Some first results of structure calculations for exotic nuclei are showing the importance of the $\delta$-dynamics for the stability conditions of drip-line nuclei [15, 16].

Aim of this paper is to present, within a $R M F$ approach, the main expected effects of the $\delta$-field on symmetry properties of the nuclear system, from EOS to $n, p$-mass splitting, and in particular on the nuclear response in unstable regions. We remind that the study of symmetric and asymmetric nuclear matter properties under extreme conditions is of great relevance for the understanding of the nuclear interaction in the medium. In particular a liquid-gas phase transition may occur in warm and dilute matter produced in heavy-ion collisions (multifragmentation events). This is one of the interesting open problems in theoretical and experimental nuclear physics. Isospin effects on heavy ion collisions (fragmentation and collective flows) have been largely discussed in recent years, see the reviews in 17, 18]. In this work we will try to offer a unified picture of the $\delta$-meson exchange influence on nuclear structure and dynamics.

In order to simplify the analysis and to pin down the most direct effects of the $\delta$ contributions we will follow a quite reduced version of the $R M F$ approach, yet including all the isoscalar $(\sigma, \omega)$ and isovector $(\delta, \rho)$ fields. Nonlinear self-interaction terms are introduced only in the isoscalar scalar $\sigma$-channel, essential in order to get right incompressibility parameter at normal density $\rho_{0}$ [19,20]. Such approximation scheme of minimal self-interacting terms is actually physically well justified in the baryon density range of interest here, roughly up to $2.5 \rho_{0}$ [21]. Moreover it is consistent with the $R M F$ assumptions of neglecting retardation and finite range effects in the field 
dynamics.

We like to stress the presence of several points of interest on the isovector channel results in this baryon density region [24:

- The slope of the symmetry term, the Symmetry Pressure, just below $\rho_{0}$ is of relevant importance for nuclear structure, being directly linked to the thickness of the neutron skin in n-rich (stable and/or unstable) nuclei, see the recent discussions in 25 27. Jointly to the $n-p$ effective masses this information appears essential for the stability of drip-line nuclei 15,16 .

Dissipative reaction mechanisms with asymmetric ions seem to be also quite sensitive to the same quantity [28,29].

- The nature of chemical instabilities and in general critical properties of warm and dilute asymmetric nuclear matter 11, 30 33.

- Isospin effects on the dynamics of heavy ion collisions at intermediate energies, see [18]. Reaction studies represent a very sensitive tool to test transport properties of symmetry effects, $n, p$ chemical potentials and effective masses [34 36].

In this paper, we first determine the model parameters by fitting the properties of the symmetric and asymmetric nuclear matter at $T=0$. We then extend the investigation to finite temperature. In particular we derive the new boundary regions for mechanical and chemical instabilities and their dependence on the various mesons entering in the theory.

This paper is organized as follows. In Sec. 2, the equation of state for nuclear matter at finite temperature is derived. Symmetry energies and effective masses are discussed in Sec.3, where the neutron matter EOS is also evaluated. The mechanical and chemical instabilities are studied in Sec. 4. General comments as well as a summary of the main conclusions are presented in the last Section.

\section{EQUATION OF STATE FOR NUCLEAR MATTER AT FINITE TEMPERATURE}

The starting point is the relativistic Lagrangian density of an interacting many-particle system consisting of nucleons, isoscalar (scalar $\sigma$, vector $\omega$ ) and isovector (scalar $\delta$, vector $\rho$ ) mesons:

$$
\begin{aligned}
\mathcal{L}= & \bar{\psi}\left[i \gamma_{\mu} \partial^{\mu}-\left(M_{N}-g_{\sigma} \phi-g_{\delta} \vec{\tau} \cdot \vec{\delta}\right)\right. \\
& \left.-g_{\omega} \gamma_{\mu} \omega^{\mu}-g_{\rho} \gamma^{\mu} \vec{\tau} \cdot \overrightarrow{b_{\mu}}\right] \psi \\
& +\frac{1}{2}\left(\partial_{\mu} \phi \partial^{\mu} \phi-m_{\sigma}^{2} \phi^{2}\right)-U(\phi)+\frac{1}{2} m_{\omega}^{2} \omega_{\mu} \omega^{\mu}
\end{aligned}
$$

$$
\begin{aligned}
& +\frac{1}{2} m_{\rho}^{2} \overrightarrow{b_{\mu}} \cdot \overrightarrow{b^{\mu}}+\frac{1}{2}\left(\partial_{\mu} \vec{\delta} \cdot \partial^{\mu} \vec{\delta}-m_{\delta}^{2} \overrightarrow{\delta^{2}}\right) \\
& -\frac{1}{4} F_{\mu \nu} F^{\mu \nu}-\frac{1}{4} \vec{G}_{\mu \nu} \vec{G}^{\mu \nu},
\end{aligned}
$$

Minimal self-interacting terms, as discussed in the introduction, are included only in the $\sigma$-channel.

Here $\phi$ is the $\sigma$-meson field, $\omega_{\mu}$ the $\omega$-meson field, $\overrightarrow{b_{\mu}}$ the charged $\rho$ meson field, $\vec{\delta}$ the isovector scalar field of the $\delta$-meson. We define $F_{\mu \nu} \equiv \partial_{\mu} \omega_{\nu}-\partial_{\nu} \omega_{\mu}, \vec{G}_{\mu \nu} \equiv$ $\partial_{\mu} \overrightarrow{b_{\nu}}-\partial_{\nu} \overrightarrow{b_{\mu}} \cdot U(\phi)$ is the nonlinear potential of the $\sigma$ meson : $U(\phi)=\frac{1}{3} a \phi^{3}+\frac{1}{4} b \phi^{4}$.

The field equations in mean field approximation $(R M F)$ are:

$$
\begin{array}{r}
\left(i \gamma_{\mu} \partial^{\mu}-\left(M_{N}-g_{\sigma} \phi-g_{\delta} \tau_{3} \delta_{3}\right)-g_{\omega} \gamma^{0} \omega_{0}\right. \\
\left.-g_{\rho} \gamma^{0} \tau_{3} b_{0}\right) \psi=0 \\
m_{\sigma}^{2} \phi+a \phi^{2}+b \phi^{3}=\bar{\psi} \psi=g_{\sigma} \rho_{S} \\
m_{\omega}^{2} \omega_{0}=g_{\omega} \bar{\psi} \gamma^{0} \psi=g_{\omega} \rho_{B} \\
m_{\rho}^{2} b_{0}=g_{\rho} \bar{\psi} \gamma^{0} \tau_{3} \psi=g_{\rho} \rho_{B 3} \\
m_{\delta}^{2} \delta_{3}=g_{\delta} \bar{\psi} \tau_{3} \psi=g_{\delta} \rho_{S 3},
\end{array}
$$

where $\rho_{B 3}=\rho_{B p}-\rho_{B n}$ and $\rho_{S 3}=\rho_{S p}-\rho_{S n}, \rho_{B}$ and $\rho_{S}$ being the baryon and the scalar densities, respectively.

Neglecting the derivatives of mesons fields, in the mean field approximation the energy-momentum tensor is given by

$$
\begin{array}{r}
T_{\mu \nu}=i \bar{\psi} \gamma_{\mu} \partial_{\nu} \psi+\left[\frac{1}{2} m_{\sigma}^{2} \phi^{2}+U(\phi)+\frac{1}{2} m_{\delta}^{2} \overrightarrow{\delta^{2}}\right. \\
\left.-\frac{1}{2} m_{\omega}^{2} \omega_{\lambda} \omega^{\lambda}-\frac{1}{2} m_{\rho}^{2} \overrightarrow{b_{\lambda}} \overrightarrow{b^{\lambda}}\right] g_{\mu \nu} .
\end{array}
$$

The properties of nuclear matter at finite temperature are described by the thermodynamic potential $\Omega$. ¿From statistical mechanics for a system in a volume $\mathrm{V}$ we define $\Omega=-p V=-\frac{1}{\beta} \ln Z$ [2], where $\beta$ the inverse of temperature, $\beta=1 / \mathrm{T}$, and $Z$ is the grand partition function given by $Z=\operatorname{Tr}\left[e^{-\beta\left(\hat{H}-\Sigma_{i}\left(\mu_{i} \hat{B}_{i}\right)\right)}\right]$. Here $\hat{H}$ is the hamiltonian operator, $\hat{B}_{i}$ and $\mu_{i}$ are respectively nucleon number operators and thermodynamic chemical potentials, $(i=p, n)$. The equation of state for nuclear matter at finite temperature can be obtained from the thermodynamic potential $\Omega$.

The energy density has the form

$$
\begin{array}{r}
\epsilon=\sum_{i=n, p} 2 \int \frac{\mathrm{d}^{3} k}{(2 \pi)^{3}} E_{i}^{\star}(k)\left(n_{i}(k)+\bar{n}_{i}(k)\right)+\frac{1}{2} m_{\sigma}^{2} \phi^{2} \\
+U(\phi)+\frac{1}{2} m_{\omega}^{2} \omega_{0}^{2}+\frac{1}{2} m_{\rho}^{2} b_{0}^{2}+\frac{1}{2} m_{\delta}^{2} \delta_{3}^{2},
\end{array}
$$

and the pressure is

$$
\begin{array}{r}
P=\sum_{i=n, p} \frac{2}{3} \int \frac{\mathrm{d}^{3} k}{(2 \pi)^{3}} \frac{k^{2}}{E_{i}^{\star}(k)}\left(n_{i}(k)+\bar{n}_{i}(k)\right)-\frac{1}{2} m_{\sigma}^{2} \phi^{2} \\
-U(\phi)+\frac{1}{2} m_{\omega}^{2} \omega_{0}^{2}+\frac{1}{2} m_{\rho}^{2} b_{0}^{2}-\frac{1}{2} m_{\delta}^{2} \delta_{3}^{2},
\end{array}
$$


where $E_{i}^{\star}=\sqrt{k^{2}+M_{i}^{\star 2}}$. The nucleon effective masses are defined as

$$
M_{i}^{\star}=M_{N}-g_{\sigma} \phi \mp g_{\delta} \delta_{3} \quad(- \text { proton },+ \text { neutron }) .
$$

The $n_{i}(k)$ and $\bar{n}_{i}(k)$ in Eqs. (4)-(5) are the fermion and antifermion distribution functions for protons $(i=p)$ and neutrons $(i=n)$ :

$$
n_{i}(k)=\frac{1}{1+\exp \left\{\left(E_{i}^{\star}(k)-\mu_{i}^{\star}\right) / T\right\}},
$$

and

$$
\bar{n}_{i}(k)=\frac{1}{1+\exp \left\{\left(E_{i}^{\star}(k)+\mu_{i}^{\star}\right) / T\right\}} .
$$

The effective chemical potentials $\mu_{i}^{\star}$ are given in terms of the vector meson mean fields

$$
\mu_{i}=\mu_{i}^{\star}-g_{\omega} \omega_{0} \mp g_{\rho} b_{0} \quad(- \text { proton },+ \text { neutron }),
$$

where $\mu_{i}$ are the thermodynamical chemical potentials $\mu_{i}=\partial \epsilon / \partial \rho_{i}$. At zero temperature they reduce to the Fermi energies $E_{F i} \equiv \sqrt{k_{F i}^{2}+M_{i}^{\star 2}}$.

The same results can be directly obtained from the expectation value of the energy-momentum tensor, showing the thermodynamical consistency of the mean field approximation [2].

By using the field equations for mesons, the equations of state for thermal matter can be rewritten as

$$
\begin{array}{r}
\epsilon=\sum_{i=n, p} 2 \int \frac{\mathrm{d}^{3} k}{(2 \pi)^{3}} E_{i}^{\star}(k)\left(n_{i}(k)+\bar{n}_{i}(k)\right)+\frac{1}{2} m_{\sigma}^{2} \phi^{2} \\
+U(\phi)+\frac{g_{\omega}^{2}}{2 m_{\omega}^{2}} \rho_{B}^{2}+\frac{g_{\rho}^{2}}{2 m_{\rho}^{2}} \rho_{B 3}^{2}+\frac{g_{\delta}^{2}}{2 m_{\delta}^{2}} \rho_{S 3}^{2},
\end{array}
$$

and

$$
\begin{array}{r}
P=\sum_{i=n, p} \frac{2}{3} \int \frac{\mathrm{d}^{3} k}{(2 \pi)^{3}} \frac{k^{2}}{E_{i}^{\star}(k)}\left(n_{i}(k)+\bar{n}_{i}(k)\right)-\frac{1}{2} m_{\sigma}^{2} \phi^{2} \\
-U(\phi)+\frac{g_{\omega}^{2}}{2 m_{\omega}^{2}} \rho_{B}^{2}+\frac{g_{\rho}^{2}}{2 m_{\rho}^{2}} \rho_{B 3}^{2}-\frac{g_{\delta}^{2}}{2 m_{\delta}^{2}} \rho_{S 3}^{2} .
\end{array}
$$

We remind that the baryon densities $\rho_{B}$ are given by ( $\gamma$ is the spin/isospin multiplicity)

$$
\rho_{B}=\gamma \int \frac{\mathrm{d}^{3} k}{(2 \pi)^{3}}(n(k)-\bar{n}(k)),
$$

while the scalar densities $\rho_{S}$ are

$$
\rho_{S}=\gamma \int \frac{\mathrm{d}^{3} k}{(2 \pi)^{3}} \frac{M^{\star}}{E^{\star}}(n(k)+\bar{n}(k)) .
$$

At the temperatures of interest here the antybaryon contributions are actually negligible.
In order to study the asymmetric nuclear matter, we introduce an asymmetry parameter $\alpha$ defined as $\alpha=$ $\left(\rho_{B n}-\rho_{B p}\right) / \rho_{B}=\frac{N-Z}{A}$.

The energy density and pressure for symmetric and asymmetric nuclear matter at finite temperature and the $n, p$ effective masses can be calculated self-consistently from Eqs. (6) to (13) just in terms of the four boson coupling constants, $f_{i} \equiv\left(\frac{g_{i}^{2}}{m_{i}^{2}}\right), i=\sigma, \omega, \rho, \delta$ and the two parameters of the $\sigma$ self-interacting terms, $A \equiv \frac{a}{g_{\sigma}^{3}}$ and $B \equiv \frac{b}{g_{\sigma}^{4}}$.

The isoscalar meson parameters are fixed from symmetric nuclear matter properties at $T=0$ : saturation density $\rho_{0}=0.16 \mathrm{fm}^{-3}$, binding energy $E / A=$ $-16 \mathrm{MeV}$, nucleon effective mass $M^{\star}=0.75 M_{N}\left(M_{N}=\right.$ $939 \mathrm{MeV}$ ) and incompressibility $K_{V}=240 \mathrm{MeV}$ at $\rho_{0}$. The fitted $f_{\sigma}, f_{\omega}, A, B$ parameters are reported in Table I and have quite standard values for these minimal nonlinear $R M F$ models.

Table I. Parameter sets.

\begin{tabular}{cccc}
\hline parameter & Set I & Set II & NL3 \\
\hline$f_{\sigma}\left(\mathrm{fm}^{2}\right)$ & 10.33 & same & 15.73 \\
\hline$f_{\omega}\left(\mathrm{fm}^{2}\right)$ & 5.42 & same & 10.53 \\
\hline$f_{\rho}\left(\mathrm{fm}^{2}\right)$ & 0.95 & 3.15 & 1.34 \\
\hline$f_{\delta}\left(\mathrm{fm}^{2}\right)$ & 0.00 & 2.50 & 0.00 \\
\hline$A\left(\mathrm{fm}^{-1}\right)$ & 0.033 & same & -0.01 \\
\hline$B$ & -0.0048 & same & -0.003 \\
\hline
\end{tabular}

In the table we report also the $N L 3$ parametrization, widely used in nuclear structure calculations 画. We remind that the $N L 3$-saturation properties for symmetric matter are chosen as $\rho_{0}=0.148 \mathrm{fm}^{-3}, M^{\star}=0.6 M_{N}$, $K_{V}=271.8 \mathrm{MeV}$ and the symmetry parameter (see next section) is $a_{4}=37.4 \mathrm{MeV}$.

The corresponding phase diagram (pressure isotherms) is shown in Fig.1. The local minimum disappears at the critical temperature $\mathrm{T}_{\mathrm{c}}$, determined by $\left.\frac{\partial P}{\partial \rho}\right|_{\mathrm{T}_{c}}=$ $\left.\frac{\partial^{2} P}{\partial \rho^{2}}\right|_{\mathrm{T}_{c}}=0$. The obtained critical temperature of symmetric matter is $\mathrm{T}_{\mathrm{c}}=15.86 \mathrm{MeV}$. 


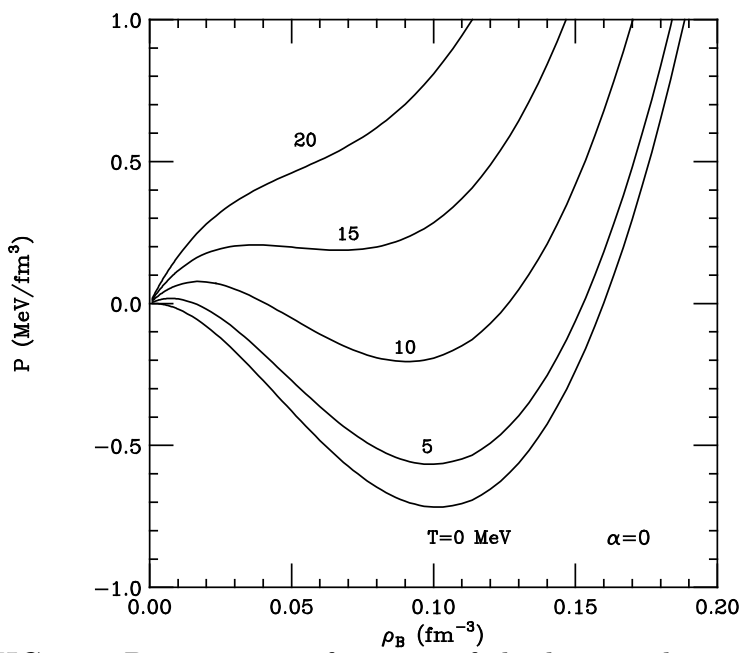

FIG. 1. Pressure as a function of the baryon density for symmetric nuclear matter $(\alpha=0)$ at different temperatures.

\section{THE SYMMETRY ENERGY}

Symmetry properties of asymmetric nuclear matter are univocally fixed from the coupling constants of the isovector channels [37. Experimentally we have just one relatively well known quantity, the bulk symmetry energy $a_{4}$ of the Weiszaecker mass formula, in the range $30-35 \mathrm{MeV}$. In all the RMF models with only the isovector $\rho$ meson this assigns the $f_{\rho}$ constant, that gives also the "slope" of the symmetry term (the Symmetry Pressure) 110], apart small non-linear contributions [23].

When we further include the $\delta$-meson, from the $a_{4}$ value we will fix only a combination of the two coupling constants, while the $\delta$ itself will imply interesting new contributions to the slope and curvature of the symmetry energy and a neutron-proton effective mass splitting. These will be the main points discussed in the following. We note that most results are analytical and this will largely improve the physical understanding of the effects due to the coupling to the $\delta$ isovector scalar channel.

The symmetry energy in asymmetric $N M$ is defined from the expansion of the energy per nucleon $E\left(\rho_{B}, \alpha\right)$ in terms of the asymmetry parameter

$$
\begin{aligned}
E\left(\rho_{B}, \alpha\right) \equiv \frac{\epsilon\left(\rho_{B}, \alpha\right)}{\rho_{B}}=E\left(\rho_{B}\right)+ & E_{\text {sym }}\left(\rho_{B}\right) \alpha^{2} \\
& +O\left(\alpha^{4}\right)+\ldots
\end{aligned}
$$

and so in general

$$
\left.E_{\text {sym }} \equiv \frac{1}{2} \frac{\partial^{2} E\left(\rho_{B}, \alpha\right)}{\partial \alpha^{2}}\right|_{\alpha=0}=\left.\frac{1}{2} \rho_{B} \frac{\partial^{2} \epsilon}{\partial \rho_{B 3}^{2}}\right|_{\rho_{B 3}=0}
$$

\section{The Bulk Symmetry Parameter}

The bulk symmetry parameter $a_{4}$ is just the value of $E_{\text {sym }}$ corresponding to the normal density $\rho_{B}=\rho_{0}$, at
$T=0$. Then we discuss first the case at zero temperature, in order to fix the isovector coupling constants. ¿From Eqs. $(10,15)$ we can easily get an explicit expression for the symmetry energy 12.

$$
E_{\text {sym }}\left(\rho_{B}\right)=\frac{1}{6} \frac{k_{F}^{2}}{E_{F}}+\frac{1}{2} f_{\rho} \rho_{B}-\frac{1}{2} f_{\delta} \frac{M^{\star 2} \rho_{B}}{E_{F}^{2}\left[1+f_{\delta} A\left(k_{F}, M^{\star}\right)\right]}
$$

where $k_{F}$ is the nucleon Fermi momentum corresponding to $\rho_{B}, E_{F} \equiv \sqrt{\left(k_{F}^{2}+M^{\star 2}\right)}$ and $M^{\star}$ is the effective nucleon mass in symmetric $N M, M^{\star}=M_{N}-g_{\sigma} \phi$.

The integral

$$
A\left(k_{F}, M^{\star}\right) \equiv \frac{4}{(2 \pi)^{3}} \int d^{3} k \frac{k^{2}}{\left(k^{2}+M^{\star^{2}}\right)^{3 / 2}}
$$

has a simple analytical structure which makes quite transparent the effect of the $\delta$-meson on the symmetry energy. With some algebra we can get

$$
A\left(k_{F}, M^{\star}\right)=3\left(\frac{\rho_{S}}{M^{\star}}-\frac{\rho_{B}}{E_{F}}\right)
$$

making use of

$$
\begin{array}{r}
\rho_{S}(T=0)=\frac{M^{\star}}{\pi^{2}}\left[k_{F} E_{F}-M^{\star 2} \ln \left(\frac{k_{F}+E_{F}}{M^{\star}}\right)\right] \\
\rho_{B}(T=0)=\frac{2}{3 \pi^{2}} k_{F}^{3}
\end{array}
$$

Expanding the scalar density in terms of $\left(k_{F} / M^{*}\right)^{2}$ [2], the quantity Eq.(18) can be written as

$$
A\left(k_{F}, M^{\star}\right)=\frac{3 \rho_{B}}{M^{*}}\left[\frac{1}{5}\left(\frac{k_{F}}{M^{*}}\right)^{2}-\frac{3}{14}\left(\frac{k_{F}}{M^{*}}\right)^{4}-\frac{5}{24}\left(\frac{k_{F}}{M^{*}}\right)^{6}+\ldots\right],
$$

that can be used to derive a similar expansion for the symmetry energy eq.(16).

We see that $A\left(k_{F}, M^{\star}\right)$ is certainly very small at low densities, below $\rho_{0}$. It can be still neglected up to a baryon density $\rho_{B} \simeq 3 \rho_{0}$, where it reaches the value $0.045 \mathrm{fm}^{-2}$ (with our symmetric $N M$ parameters), i.e. a correction of about $10 \%$ in the denominator of Eq.(16).

Then in the density range of interest here we can use, at the leading order, the much simpler form of the symmetry energy, extremely nice in order to get the main qualitative new features due to the $\delta$-meson coupling:

$$
E_{\text {sym }}\left(\rho_{B}\right)=\frac{1}{6} \frac{k_{F}^{2}}{E_{F}}+\frac{1}{2}\left[f_{\rho}-f_{\delta}\left(\frac{M^{\star}}{E_{F}}\right)^{2}\right] \rho_{B}
$$

We see that, when the $\delta$ is included, the observed $a_{4}$ value actually assigns the combination $\left[f_{\rho}-f_{\delta}\left(\frac{M^{\star}}{E_{F}}\right)^{2}\right]$ of the $(\rho, \delta)$ coupling constants. If $f_{\delta} \neq 0$ we have to increase the $\rho$-coupling (see Fig.1 of [12]). In our calculations we use the value $a_{4}=30.5 \mathrm{MeV}$. In Table I the Set I corresponds to $f_{\delta}=0$. In the Set II $f_{\delta}$ is chosen as 
$2.5 \mathrm{fm}^{2}$. Although this value is relatively well justified, [39], we stress that aim of this work is just to show the main qualitative new effects of the $\delta$-coupling.

In order to have the same $a_{4}$ we must increase the $\rho$ coupling constant of a factor three, up to $f_{\rho}=3.15 \mathrm{fm}^{2}$. From the structure of Eqs. $(16,20)$ it is clear that there is a connection between the scalar field (isoscalar, $\sigma$, and isovector, $\delta$ ) contributions, since both are acting on effective masses. A strong $\sigma$-coupling (smaller $M^{*}$, e.g. see NL3 parametrization) can compensate a strong $\delta$ coupling. In our evaluation we keep fixed the $\sigma$ parameters $\left(f_{\sigma}, A, B\right)$ leading to $M^{*}=0.75 M_{N}$. In any case a larger value of $E_{\text {sym }}$ at high baryon densities, due to the relativistic mechanism discussed in the following, see Fig.2, will be always present when the $\delta$ is included.

At subnuclear densities, $\rho_{B}<\rho_{0}$, in both cases, $(\rho)$ and $(\rho+\delta)$, from Eq. (20) we have an almost linear dependence of $E_{\text {sym }}$ on the baryon density, since $M^{\star} \simeq E_{F}$ as a good approximation. Around and above $\rho_{0}$ we expect a steeper increase in the $(\rho+\delta)$ case since $M^{\star} / E_{F}$ is decreasing, see Fig.2.

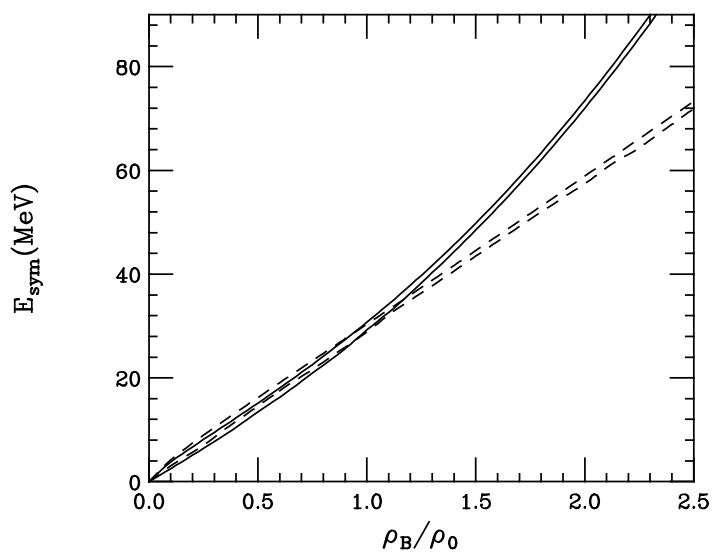

FIG. 2. Total (kinetic + potential) symmetry energy as a function of the baryon density. Dashed lines: $(\rho)$. Solid lines: $(\rho+\delta)$. Upper curves: zero temperature. Lower curves: $T=8 \mathrm{MeV}$

This is an interesting aspect to look at more in detail since it actually represents a general relativistic effect due to the coupling to scalar mesons. In some sense it is the equivalent, in the isovector channel $(\delta$ vs. $\rho$ ), i.e. for the symmetry energy, of the saturation mechanism we have in the isoscalar channel ( $\sigma$ vs. $\omega)$ for the symmetric matter.

The scalar charged $\delta$-meson, like the neutral $\sigma$, is acting on the nucleon effective masses, introducing a $n, p$ splitting, see Eq.(6). This causes a negative contribution to $E_{\text {sym }}$, Eqs.(16),(21), since it reduces the gap between $n, p$ Fermi energies due to the different Fermi momenta in asymmetric $N M$. In fact in a n-rich matter the neutron
Fermi momentum increases while the neutron effective mass decreases, see Eq.(6) and Fig.6 (the opposite for protons).

Such negative $\delta$ contribution is reduced at high densities due to the "Lorentz contraction" factor $\left(M^{\star} / E_{F}\right)^{2}$, Eqs.(16) and (20), that in general gives the attenuation of the scalar interactions with increasing baryon density.

We stress the consistent picture of a symmetry energy built from the balance of scalar (attractive) and vector (repulsive) contributions, with the scalar channel becoming weaker with increasing baryon density. This is indeed the isovector counterpart of the saturation mechanism occurring in the isoscalar channel for the symmetric nuclear matter. From such scheme we get a further strong fundamental support for the introduction of the $\delta$-coupling in the symmetry energy evaluation.

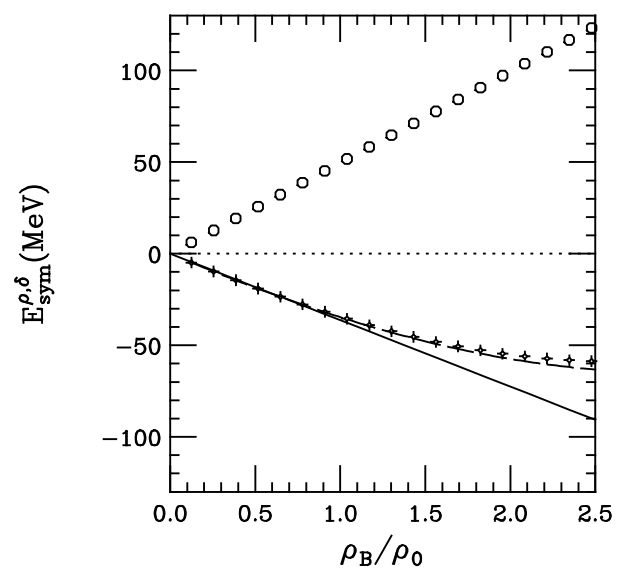

FIG. 3. $\rho$ - (open circles) and $\delta$ - (crosses) contributions to the symmetry energy, second and third terms of Eq.(16). The dashed line is the approximate $\delta$-contribution of Eq.(20), see text. The solid line is the linear extrapolation of the low density behaviour, plotted to guide the eye.

In Fig.3, using our parametrizations, we quantitatively show the interplay of the two contributions ( $\rho$ and $\delta$ ) to $E_{\text {sym }}$, second and third terms of Eqs.(16),(20), as a function of the baryon density. The crosses (negative $\delta$ contribution) follow a linear behaviour up to roughly $\rho_{0}$ and then they tend to saturate due to the Lorentz contraction factor. In correspondence we will see the increase of the total $E_{\text {sym }}$ shown in Fig.2. We note the accuracy of the approximate $\delta$ contribution given by Eq.(20), dashed line in Fig.3.

In Fig. 4 we show the Equation of State (energy per nucleon) for pure neutron matter $(\alpha=1)$ obtained with the two parameter Sets, I $(\rho)$ and II $(\rho+\delta)$.

The values are in good agreement with recent nonrelativistic Quantum-Monte-Carlo variational calculations with realistic $2-$ and 3 -body forces [41]. The inclusion of the $\delta$-coupling leads to a larger repulsion at 
baryon densities roughly above $1.5 \rho_{0}$. This could be of interest for the structure of neutron stars and the possibility of a transition to new forms of nuclear matter.

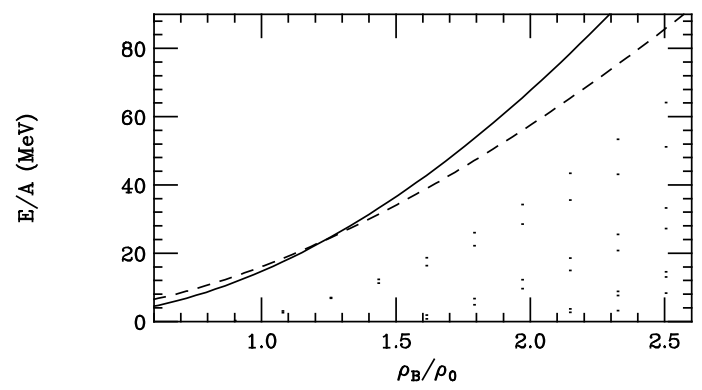

FIG. 4. EOS for pure neutron matter. Dashed line: $(\rho)$. Solid line: $(\rho+\delta)$.

¿From the previous analysis we expect to see also interesting $\delta$-effects on the slope (symmetry pressure) and curvature (symmetry incompressibility) of the symmetry energy around $\rho_{0}$, of relevant physical meaning. This will be the main subject of the following discussion.

\section{Symmetry Pressure and Symmetry Incompressibility}

In order to have a quantitative evaluation of the effect on the slope and curvature, and also to compare with other estimates, we use the expansion of the symmetry energy around $\rho_{0} 42,43$

$$
E_{\text {sym }}\left(\rho_{B}\right)=a_{4}+\frac{L}{3}\left(\frac{\rho_{B}-\rho_{0}}{\rho_{0}}\right)+\frac{K_{s y m}}{18}\left(\frac{\rho_{B}-\rho_{0}}{\rho_{0}}\right)^{2}+\ldots
$$

with $L$ and $K_{\text {sym }}$ respectively related to slope and curvature of the symmetry energy at $\rho_{0}$

$$
\begin{array}{r}
L=\left.3 \rho_{0}\left(\frac{\partial E_{\text {sym }}}{\partial \rho_{B}}\right)\right|_{\rho_{B}=\rho_{0}} \quad \text { and } \\
K_{\text {sym }}=\left.9 \rho_{0}^{2}\left(\frac{\partial^{2} E_{\text {sym }}}{\partial \rho_{B}^{2}}\right)\right|_{\rho_{B}=\rho_{0}}
\end{array}
$$

¿From Eq.(20) we get a potential contribution to the density variation of $E_{\text {sym }}$ given by (after some algebra)

$$
\begin{array}{r}
\left.\frac{\partial E_{s y m}}{\partial \rho_{B}}\right|_{p o t}=\frac{1}{2}\left[f_{\rho}-f_{\delta}\left(\frac{M^{\star}}{E_{F}}\right)^{2}\right]+ \\
f_{\delta}\left(\frac{M^{\star} k_{F}}{E_{F}^{2}}\right)^{2}\left[\frac{1}{3}-\frac{\rho_{B}}{M^{\star}} \frac{\partial M^{\star}}{\partial \rho_{B}}\right]
\end{array}
$$

Around normal density $\rho_{0}$ the first term is fixed by the $a_{4}$ value and so we get a net increase of the slope, due to the $\delta$, given by the second term (always positive since $\left.\left(\partial M^{\star} / \partial \rho_{B}\right)<0\right)$.

¿From the first term of Eq.(23), with our parametrization, we get a potential contribution to $L$ of $45 \mathrm{MeV}$ (this is actually fixed by the $a_{4}$ value) and a genuine $\delta$ extra contribution of about $20 \mathrm{MeV}$ from the second term. When we include also the kinetic part (from the first term of Eq.(20)) we have a total slope parameter going from $L(\rho)=+84 M e V$ to $L(\rho+\delta)=+103 M e V$.

We note again that the slope parameter, or equivalently the Symmetry Pressure $P_{\text {sym }} \equiv \rho_{0} L / 3$, is of great importance for structure properties, being linked to the thickness of the neutron skin in n-rich (stable and/or unstable) nuclei [24 27], and to the assessment of the dripline 16]. Moreover the same parameter gives an estimate of the shift of the saturation density with asymmetry (at the lowest order in $\alpha^{2}$ )

$$
\Delta \rho_{0}(\alpha)=-\frac{3 \rho_{0} L}{K_{V}(\alpha=0)} \alpha^{2}
$$

that can be easily obtained from a linear expansion around the symmetric value $\rho_{0}(\alpha=0) 29$.

Eq.(24) has a simple physical meaning: in order to compensate the symmetry pressure in asymmetric matter we have to move the zero of the $P\left(\rho_{B}\right)$ curve to lower densities. The amount of the shift will be inversely proportional to the slope, given by the incompressibility.

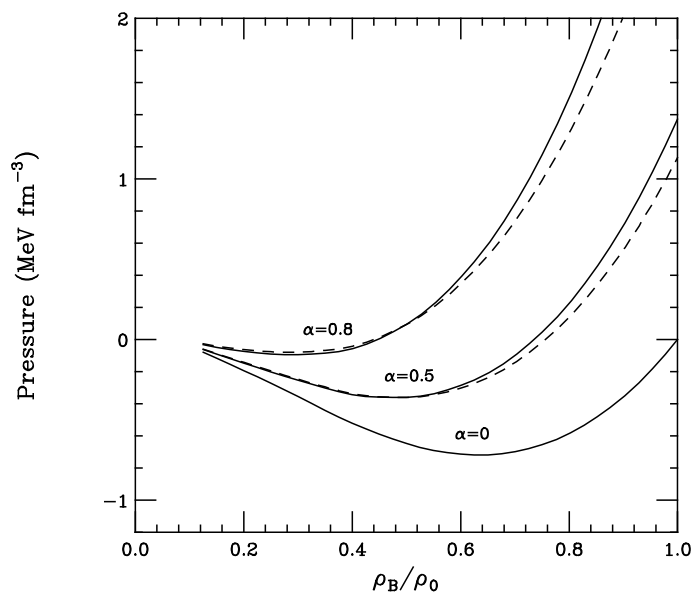

FIG. 5. Pressure as a function of the baryon density at zero temperature for various asymmetries $\alpha=0$ (bottom), 0.5 (middle), 0.8 (top). Dashed lines: $(\rho)$. Solid lines: $(\rho+\delta)$.

All that can be seen in Fig. 5 where we report the total pressure $P\left(\rho_{B}\right)$ for various asymmetries, at zero temperature, in the two cases, $\rho$-meson only (dashed lines) and $(\rho+\delta)$ (solid lines).

It is instructive to perform a similar discussion for the curvature parameter $K_{\text {sym }}$, Eq.(21). Now the potential contribution is exclusively given by the $\delta$-meson, with a definite positive sign, as we can see from the previous discussion. This will be a large effect on the total since the kinetic part is quite small [45]. If we compare with non-relativistic effective parametrizations [44,43], when we add the $\delta$-meson we move from a linear to a roughly parabolic $\rho_{B}$-dependence of the symmetry energy. 
With our parameters we pass from a $K_{\text {sym }}(\rho)=$ $+7 \mathrm{MeV}$ (only kinetic) to a $K_{\text {sym }}(\rho+\delta)=+120 \mathrm{MeV}$. So this quantity appears extremely interesting to look at experimentally, as recently suggested from reaction measurements 43. The problem is that the effect on the total incompressibility of asymmetric matter, that likely could be easier to measure, is not trivial. We can evaluate a shift of the incompressibility with asymmetry, at the same $\alpha^{2}$ order as in Eq.(24), as 42, 29

$$
\Delta K_{V}(\alpha)=\left(K_{\text {sym }}-6 L\right) \alpha^{2}
$$

So what really matters for the total incompressibility is the combination $\left(K_{\text {sym }}-6 L\right)$, with the possibility of a compensation between the two terms. Just by chance this is actually what is happening in our calculations since for the above combination we get $-497 \mathrm{MeV}$ in the case of only $\rho$ coupling, and $-504 \mathrm{MeV}$ when we add also the $\delta$. Indeed we see from Fig.5 that the slopes of the pressure around equilibrium density $\left(P\left(\rho_{0}\right)=0\right)$ are very close in the two cases, $(\rho)$ and $(\rho+\delta)$.

\section{Effective Mass Splitting}

Another qualitative new result of the $\delta$-meson coupling is the $n, p$-effective mass splitting in asymmetric matter [12], see Eq.(6). In Fig.6 we report the baryon density dependence of the $n, p$ effective masses for $\alpha=0.5,(N=$ $3 Z$ ) asymmetry, calculated with our Set II parameters, compared to the symmetric case 46 .

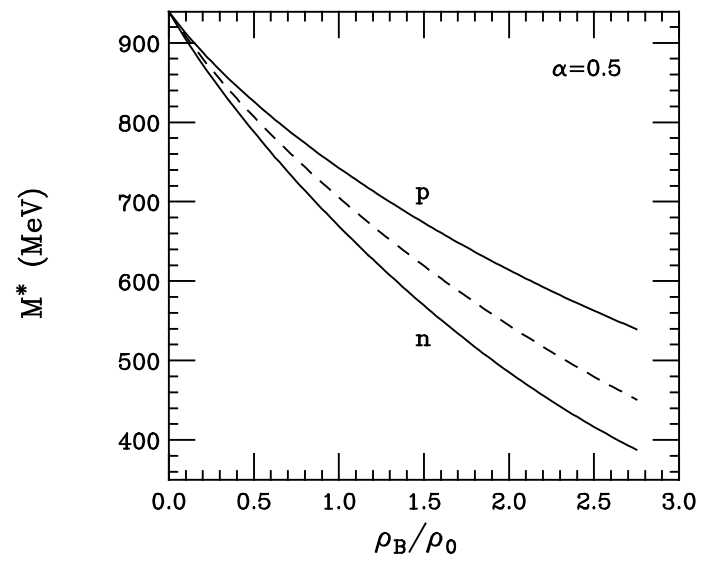

FIG. 6. Neutron, proton effective masses vs. the baryon density for $\alpha=0.5(N=3 Z)$. The dashed line corresponds to symmetric nuclear matter.

We get a splitting of the order of $15 \%$ at normal density $\rho_{0}$, and increasing with baryon density. Unfortunately from the present nuclear data we have a very small knowledge of this effect, due to the low asymmetries available.
This issue will be quite relevant in the study of dripline nuclei. Moreover we can expect important effects on transport properties ( fast particle emission, collective flows) of the dense and asymmetric $N M$ that will be reached in Radioactive Beam collisions at intermediate energies.

The sign itself of the splitting would be very instructive. As we can see from Eq.(6) in $n$-rich systems we expect a neutron effective mass always smaller than the proton one. The same is predicted from more microscopic relativistic Dirac-Brueckner calculations [15]. At variance, non-relativistic Brueckner-Hatree-Fock calculations are leading to opposite conclusions [9]4. Still in the non-relativistic picture quite contradictory results are obtained with the Skyrme effective forces. The most recent parametrizations, SLy-type 48], of Skyrme forces give the proton effective mass above the neutron one, in agreement with our calculations. Previous Skyrme-like forces, instead, yield a splitting in the opposite direction, but also show unpleasant behaviours in the spin channel (collapse of polarized neutron matter, see discussion in $48)$.

We like to note again that, as shown before, a decreasing neutron effective mass in $n$-rich matter is behind the relativistic mechanism for the symmetry energy, balance of scalar (attractive) and vector (repulsive) contributions in the isovector channel.

\section{Finite Temperature Effects}

In the temperature range of interest in this paper, below the critical temperature $T_{c}$ of the liquid-gas phase transition, of the order of $15-16 \mathrm{MeV}$ (see Fig.1), temperature effects on the symmetry properties are not expected to be large. Indeed the contributions of antifermions, that could modify all the terms with the scalar densities, see Eq.(13), are still very reduced. In Fig. 2 we present also the symmetry energies calculated at $T=8 \mathrm{MeV}$, lower solid and dashed curves. For both cases, $\rho$ and $(\rho+\delta)$ the variation is quite small. We have a reduction mainly coming from the kinetic contribution due to the smoothing of the $n, p$ Fermi distributions. We note that this result is in full agreement with relativistic Brueckner-Hartree-Fock calculations [49].

The effect is even smaller for the mass splitting, given by a difference of scalar densities. In the next section we will study in detail the phase diagram of heated asymmetric nuclear matter, focussing in particular on the instability regions. 


\section{MECHANICAL AND CHEMICAL INSTABILITIES}

Heavy-ion collisions can provide the possibility of studying equilibrated nuclear matter far away from normal conditions, i.e. to sample new regions of the $N M$ phase diagram. In particular the process of multifragmentation allows to probe dilute nuclear matter at finite temperatures, where in the symmetric case, see Fig.1, we expect to see a phase transition of first order of liquid-gas type, as suggested from the very first equations of state built with effective interactions 50 52.

The multifragmentation phenomenon may be interpreted as a signal of such a phase transition in a finite system, when the nuclear matter in the expansion phase enters the region of spinodal (mechanical) instability. Nuclear matter is however a two-component system consisting of neutrons and protons: binary systems have more complicated phase diagrams due to the new concentration degree of freedom. In particular for asymmetric nuclear matter a qualitative new feature in the liquid-gas phase transition is expected, the onset of a coupling to chemical instabilities (component separation) that will show up in a novel nature of the unstable modes, the mixture of density and charge fluctuations leading to an Isospin Distillation effect 11,30,33. Indeed equilibrium thermodynamics, as well as non-equilibrium kinetic considerations predict that an asymmetric system will separate into more symmetric larger fragments ("liquid") and into neutron-rich light fragments ("gas"). So the chemical instability can be investigated experimentally just by measuring the $\mathrm{N} / \mathrm{Z}$ ratio, or isospin content, of the fragments.

Since the effect is driven by the isospin dependent part of the nuclear equation of state, here we will look at the influence of the $\delta$ coupling on this new liquid-gas phase transition.

It is known that the stability condition of a twocomponent, $n-p$, thermodynamical system is given by

$$
\left(\frac{\partial P}{\partial \rho}\right)_{T, y}\left(\frac{\partial \mu_{p}}{\partial y}\right)_{T, P}>0
$$

where $\mathrm{P}$ is the pressure, $\mu_{p}$ is the proton chemical potential and $y$ the proton fraction $Z / A$, related to the asymmetry parameter $\alpha=1-2 y$.

Eq.(26) is equivalent to set the free energy to be a convex function in the space of the $n, p$ density oscillations, $\delta \rho_{n}, \delta \rho_{p}$. In charge symmetric matter isoscalar (total density) $\delta \rho_{n}+\delta \rho_{p}$ and isovector (concentration) $\delta \rho_{n}-\delta \rho_{p}$ oscillations are not coupled and we have two separate conditions for instability

$$
\left(\frac{\partial P}{\partial \rho}\right)_{T, y} \leq 0
$$

mechanical, i.e. vs. density oscillations (the spinodal region), and

$$
\left(\frac{\partial \mu_{p}}{\partial y}\right)_{T, P} \leq 0
$$

chemical, i.e. vs. concentration oscillations.

In asymmetric matter the isoscalar and isovector modes are coupled and the two separate inequalities do not maintain anymore a physical meaning, in the sense that they not select the nature of the instability. Inside the general condition Eq.(26) the corresponding unstable modes are a mixing of density and concentration oscillations, very sensitive to the charge dependent part of the nuclear interaction in the various instability regions [33].

In dilute asymmetric $N M$ (n-rich) the normal unstable modes for all realistic effective interactions are still isoscalarlike, i.e. in phase $n-p$ oscillations but with a larger proton component 33]. This leads to a more symmetric high density (liquid) phase everywhere under the instability line defined by Eq.(26) and consequentely to a more neutron-rich gas (Isospin Distillation). Such "chemical effect" is driven by the increasing symmetry repulsion going from low density to roughly the saturation value and so it appears quite sensitive to the symmetry energy of the used effective interaction at subnuclear densities. It could provide a good opportunity to differentiate the various EOS isospin dependences [53].

In this section we study the effect of the $\delta$-coupling on the instability region given by Eq.(26) in dilute asymmetric nuclear matter and on the structure of the corresponding unstable modes. We start from an identity valid for any binary thermodynamical system

$$
\begin{array}{r}
\left(\frac{\partial \mu_{p}}{\partial \rho_{p}}\right)_{T, \rho_{n}}\left(\frac{\partial \mu_{n}}{\partial \rho_{n}}\right)_{T, \rho_{p}}-\left(\frac{\partial \mu_{p}}{\partial \rho_{n}}\right)_{T, \rho_{p}}\left(\frac{\partial \mu_{n}}{\partial \rho_{p}}\right)_{T, \rho_{n}}= \\
\frac{1}{(1-y) \rho^{2}}\left(\frac{\partial P}{\partial \rho}\right)_{T, y}\left(\frac{\partial \mu_{p}}{\partial y}\right)_{T, P}
\end{array}
$$

where $\mu_{q}, \rho_{q}(q=n, p)$ are respectively neutron/proton chemical potentials and densities. From our knowledge of the chemical potential on each isotherm, Eq.(9), we can easily compute the limits of the instability region in the $T, \rho_{B}$ plane for dilute asymmetric $N M$ in the two choices, without and with the $\delta$ meson.

The results are reported in Fig.7. The $\delta$ inclusion (solid lines) appears not much affecting the instability limits even at relatively large asymmetry $\alpha=0.8(N \simeq 9 Z)$. We notice just a small reduction and a shift to the left (lower densities) of the whole region: this can be understood in terms of the larger symmetry repulsion, see Fig.3. 


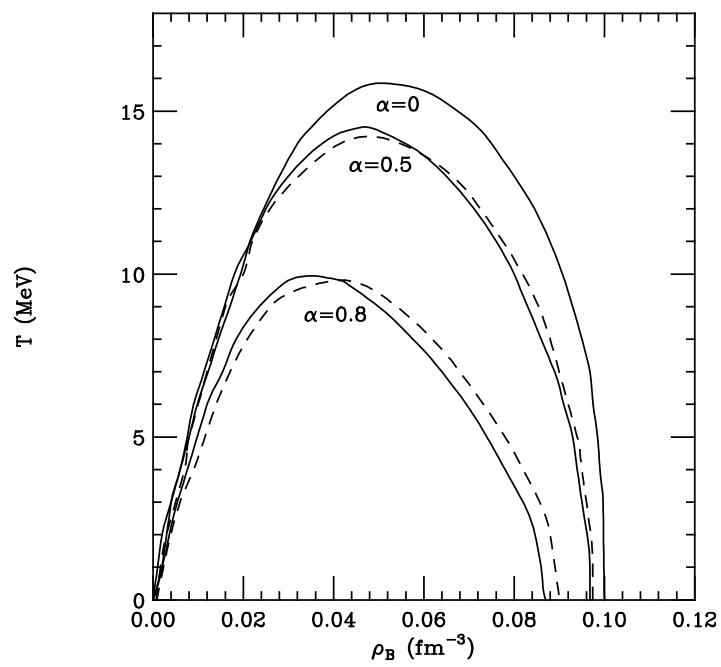

FIG. 7. Limits of the instability region in the $T, \rho_{B}$ plane for various asymmetries. Dashed lines: $(\rho)$. Solid lines: $(\rho+\delta)$ [for $\alpha \neq 0$ ].

We can understand the relatively small $\delta$ effect on the stability border just remembering that for low densities, well below $\rho_{0}$, the symmetry term has roughly the same linear behaviour in both $(\rho)$ and $(\rho+\delta)$ schemes, fixed by the $a_{4}$ parameter (see the discussion after Eq.(20)). Only for very large asymmetries it appears relatively easier in the $\delta$ case to be in the stable liquid phase.

A rather larger difference can be seen in the behaviour of the quantity Eq.(26) inside the instability region. This is plotted in Fig.8 for various asymmetries at zero temperature and in Fig.9 at various temperatures for a fixed asymmetry $\alpha=0.5(N=3 Z)$. The solid curves (with $\delta$-coupling) are systematically above the dashed ones, signature of a weaker instability.

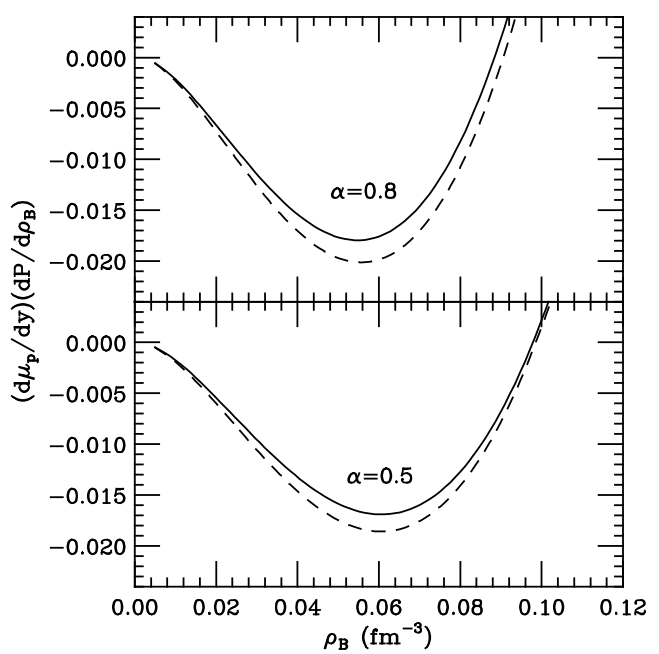

FIG. 8. The quantity Eq.(26) inside the instability region at $T=0$ and various asymmetries. Dashed lines: $(\rho)$. Solid lines: $(\rho+\delta)$.

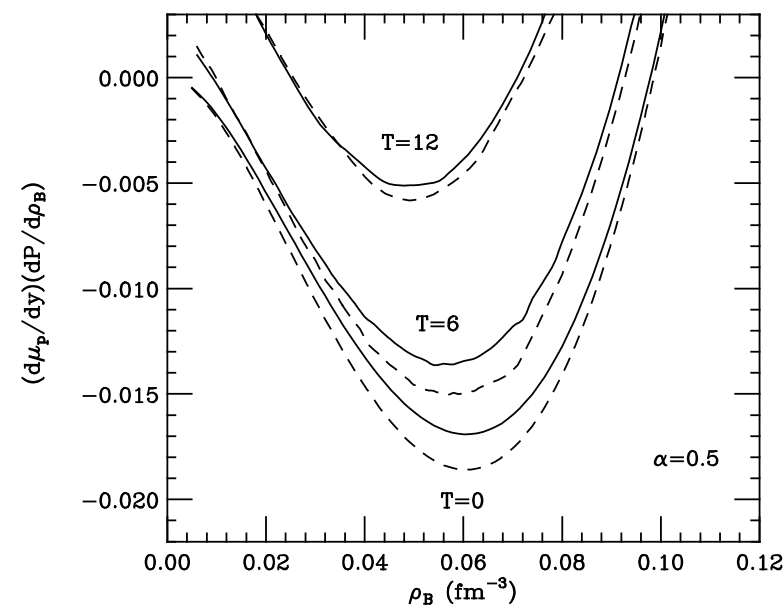

FIG. 9. The same as in Fig.8 at various temperatures and fixed asymmetry $\alpha=0.5$. Dashed lines: $(\rho)$. Solid lines: $(\rho+\delta)$.

In order to better understand the origin of such effect we study also the structure of the corresponding unstable modes. We follow the Landau dispersion relation approach to small amplitude oscillations in Fermi liquids [54,55, 31, 32]. For a two component $(n, p)$ matter the interaction is characterized by the Landau parameters $F_{0}^{q, q^{\prime}},\left(q, q^{\prime}\right)=(n, p)$ defined as

$$
N_{q}(T) \frac{\partial \mu_{q}}{\partial \rho_{q^{\prime}}} \equiv \delta_{q, q^{\prime}}+F_{0}^{q, q^{\prime}}
$$

where $N_{q}(T)$ represents the single particle level density at the Fermi energy. At zero temperature it has the simple form

$$
N_{q}=\frac{k_{F q} E_{F q}^{*}}{\pi^{2}}, \quad q=n, p .
$$

In the symmetric case $\left(F_{0}^{n n}=F_{0}^{p p}, F_{0}^{n p}=F_{0}^{p n}\right)$, the Eqs. $(27,28)$ correspond to the two Pomeranchuk instability conditions

$$
\begin{gathered}
F_{0}^{s}=F_{0}^{n n}+F_{0}^{n p}<-1 \quad \text { mechanical } \\
F_{0}^{a}=F_{0}^{n n}-F_{0}^{n p}<-1 \quad \text { chemical } .
\end{gathered}
$$

¿From the dispersion relations $F_{0}^{s}$ will give the properties of density (isoscalar) modes and $F_{0}^{a}$ of the concentration (isovector) modes. For asymmetric $N M$ we have some corresponding generalized Landau parameters $F_{0 g}^{s}, F_{0 g}^{a}$ which will characterize the new collective response (respectively isoscalarlike and isovectorlike). They can be expressed as fixed combinations of the $F_{0}^{q, q^{\prime}}$ for each baryon density, asymmetry and temperature. This transformation is reducing Eq.(29) to a "diagonal" form [33]

$$
\begin{aligned}
\left(1+F_{0 g}^{s}\right)\left(1+F_{0 g}^{a}\right)= & \frac{4}{(1-y) \rho^{2}}\left(\frac{N_{n} N_{p}}{N_{n}+N_{p}}\right)^{2} \\
& \times\left(\frac{\partial P}{\partial \rho}\right)_{T, y}\left(\frac{\partial \mu_{p}}{\partial y}\right)_{T, P}
\end{aligned}
$$


As already discussed in the unstable region of dilute asymmetric $N M$ we have isoscalarlike unstable modes and so $1+F_{0 g}^{s}<0$, while the combination $1+F_{0 g}^{a}$ will stay positive. In Fig.10 we report the full calculation of these two quantities in the unstable region at zero temperature, for asymmetry $\alpha=0.5$, with and without the $\delta$ coupling.

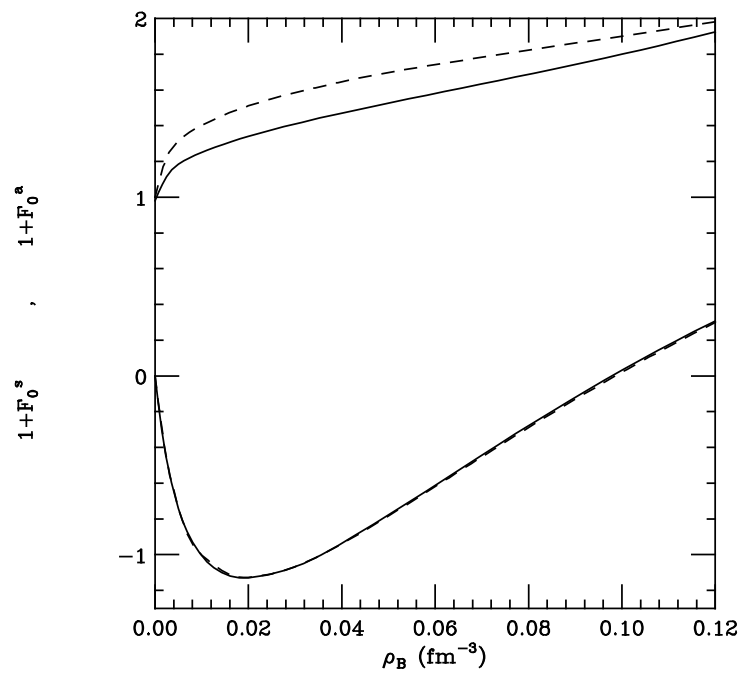

FIG. 10. Behaviour of the generalized Landau parameters inside the instability region at zero temperature and asymmetry $\alpha=0.5(N=3 Z)$. Dashed lines: $(\rho)$. Solid lines: $(\rho+\delta)$

The $\delta$ meson is almost not affecting at all the unstable mode, given by the $F_{0 g}^{s}$ parameter, and so the limits of the instability region, for $\alpha=0.5$, are not changed (see the Figs.7,8,9). We have a much larger effect on the $F_{0 g}^{a}$ parameter which describe "stable" isovectorlike modes, that actually can propagate as good zero-sound collective motions since $F_{0 g}^{a}>0$. This can be expected from the isovector nature of the $\delta$-meson. ¿From here we get the main differences seen in Figs.8,9 for the quantity Eq.(26) inside the instability region, just using Eq.(32).

Another interesting aspect of the comparison between Fig. 8 and Fig. 10 is the shift of the "maximum instability" density region. From the thermodynamical condition reported in Fig.8 it seems that the largest instability (the most negative value) is around $\rho_{B}=0.06 \mathrm{fm}^{-3}$. In fact from Fig.10 we see that the fastest unstable mode, corresponding to the most negative Pomeranchuk condition for $1+F_{0 g}^{s}$, is actually present for more dilute matter, around $\rho_{B}=0.02 \mathrm{fm}^{-3}$. This shows the importance of the linear response analysis.

Finally the fact that the $\delta$-coupling is mostly affecting the isovectorlike modes is of great interest for possible effects on the isovector Giant Dipole Resonances studied around normal density within the $R M F$ approach in asymmetric systems.

\section{CONCLUSION AND OUTLOOK}

Aim of this work has been to select genuine new effects on properties of the asymmetric nuclear matter due to the coupling to the charged $\delta$-scalar meson introduced in a minimal way in a phenomenological hadronic field theory. Simple analytical results allow to clarify the interplay between the $\rho$ and $\delta$ isovector meson contributions.

With respect to the Equation of State, when the $\delta$ is included the symmetry energy keeps an almost linear baryon density repulsive behaviour at sub-nuclear densities while it starts to move to a roughly parabolic trend above the saturation density $\rho_{0}$. Such effect comes directly from the general relativistic property of the weakening of the attractive scalar interactions at high density. It represents the equivalent in the isovector channel (interplay of $\rho$ and $\delta$ contributions) of the saturation mechanism of the symmetric $N M$ (interplay of $\omega$ and $\sigma$ contributions). All that gives a further fundamental support to the introduction of the $\delta$-channel in the symmetry energy evaluation.

Such " $\delta$ mechanism" for the symmetry energy leads to a more repulsive EOS for pure neutron matter at baryon densities roughly above $2 \rho_{0}$. This is the region where transitions to different forms of nuclear matter are expected and so the result appears quite stimulating.

We have shown in details that the new $\delta$ contributions are not negligible for the slope parameter around $\rho_{0}$ (the symmetry pressure) and absolutely essential for the curvature parameter (symmetry incompressibility). The proton-neutron effective mass splitting is also directly given by the $\delta$ coupling and appears to be of the order of $15 \%$ at normal nuclear density (for a $N=3 Z$ asymmetry).

The possibility of an experimental observation of such effects is suggested. We list here some sensitive observables:

- Neutron distributions in $n$-rich nuclei (stable and unstable), 25 27

- Assessment of drip-line stabilities [15, 16

- Bulk densities and incompressibility modulus in asymmetric nuclei [29, 42, 43]

- Transport properties in Radioactive Beam collisions at intermediate energies: dissipative mechanisms, fast nucleon emission, collective flows [18, 28, 34, 36, 44, 56

Effects on the new critical properties of warm $A N M$, mixing of mechanical and chemical instabilities and isospin distillation, are also presented. The border of the instability regions as well as the nature of the unstable fluctuations in dilute asymmetric matter are not much affected. Indeed in the low density region the symmetry 
energy has a very similar behaviour with and without the $\delta$ meson. This reduces the possibility of the observation of $\delta$-coupling effects from measurements of isospin effects in multifragmentation events in asymmetric heavy ion collisions. We note however that other fragment production mechanisms, like "neck fragmentation", are expected to be very sensitive to the symmetry potential around normal density since clusters are now formed in regions in contact with the "spectator" matter [35,36. We can predict some interesting effects on this kind of events, that actually represent a quite large part of the fragment production cross section.

¿From the study of the $\delta$ influence on the Landau parameters in the low baryon density region we note however an interesting effect on the collective response of asymmetric $N M$. While the unstable (isoscalarlike) modes are almost not modified, the isovectorlike ones, which appear to have a good stable zero-sound propagation, are quite sensitive to the introduction of the $\delta$. This suggests some importance of the $\delta$ meson in the $R M F$ description of isovector giant resonances in asymmetric nuclei.

In conclusion we would like to add a comment on the limits of the Hartree scheme used in this work, as well as in most $R M F$ calculations. Indeed it is well known that exchange terms can give contributions in the isovector scalar channel also in absence of explicit $\delta$-couplings, see ref. [38] and ref.s therein. The comparison with DiracBrueckner-Hartree-Fock results of ref. [15] and some NonLinear-Hartree-Fock calculations performed in [38], [57, are showing that an effective $\delta$ coupling parameter $f_{\delta} \simeq$ $2.5 \mathrm{fm}^{2}$, almost constant in a wide density range, is well accounting for the Fock term contributions. This makes us more confident on the reliability of the quantitative evaluation of the $\delta$ effects discussed in this paper.

\section{Acknowledgments}

One of authors (L.B.) would like to thank the LNS in Catania for the hospitality during his stay. This work was supported by the INFN of Italy and the National Natural Science Foundation of China.

[1] J.D.Walecka, Ann.Phys. (N.Y.) 83, 491 (1974).
[2] B.D.Serot and J.D.Walecka, Adv.Nucl.Phys. 16, 1 (1986); Int.J.Mod.Phys. E6, 515 (1997).

[3] Y.Sugahara and H.Toki, Nucl.Phys. A579, 557 (1994).

[4] G.A.Lalazissis, J.Konig and P.Ring, Phys.Rev. C55, 540 (1997).

[5] M.M.Sharma, A.R.Farhan and S.Mythili, Phys.Rev. C61, 054306 (2000).

[6] K.Sumiyashi and H.Toki, Astro.Phys.Jour. 422, 700 (1994).

[7] C.H.Lee, Phys.Rep. 275, 255 (1996).

[8] M.Prakash et al., Phys.Rep. 280, 1 (1997).

[9] I.Bombaci, "EOS for isospin-asymmetric nuclear matter for astrophysical applications", in [18] pp. 35-81 and ref.s therein.

[10] S.Yoshida, H.Sagawa and N.Takigawa, Phys.Rev. C58, 2796 (1998).

[11] H.Mueller and B.D.Serot, Phys.Rev. C52, 2072 (1995).

[12] S.Kubis and M.Kutschera, Phys.Lett. B399, 191 (1997).

[13] H.Shen, Y.Sugahara and H.Toki, Phys.Rev. C55, 1211 (1997).

[14] F.de Jong and H.Lenske, Phys.Rev. C57, 3099 (1998).

[15] F.Hofmann, C.M. Keil and H.Lenske, Phys.Rev. C64, 034314 (2001).

[16] J.Leja and S.Gmuca, Acta Phys.Slovaca 51, 201 (2001), and S.Gmuca, Proc. Int.Symposium on Exotic Nuclei, Baikal 2001, World Sci. in press

[17] Proceedings Nucleus-Nucleus Collisions VII, Nucl. Phys. A685, pp.1-493c (2001).

[18] Isospin Physics in Heavy Ion Collisions, Ed.s Bao-An Li and Udo Schroeder, Nova Sci.Publ. 2001, pp. 1-480.

[19] J.Boguta and A.R.Bodmer, Nucl.Phys. A292, 413 (1977).

[20] A.R.Bodmer, Nucl.Phys. A526, 703 (1991).

[21] We like to remark that in general non-linear terms in the isoscalar vector channel $\omega$ appears to be less important, see [11,22, as also confirmed the good nuclear structure results of the $N L 3$ parametrization [4. Also in the case of the isovector vector channel $\rho$ non-linear terms have been shown to have small effects 23.

[22] R.J.Furnstahl and B.D.Serot, Nucl.Phys. A671, 447 (2000).

[23] H.Mueller and B.D.Serot, Nucl.Phys. A606, 508 (1996).

[24] "It is important to put the properties of neutron matter at subnuclear densities on as firm a footing as possible, not only for astrophysical applications, but also for interpreting terrestrial experiments with coming radioactive beam faciltites", C.J.Pethick and D.G.Ravenhall, in The Lives of Neutron Stars, NATO ASI Series C Vol. 450 (1995), pp.59-70.

[25] B.A.Brown, Phys.Rev.Lett. 85, 5296 (2000).

[26] S.Typel and B.A.Brown, Phys.Rev. C64, 027302 (2001).

[27] C.J.Horowitz and J.Piekarewicz, Phys.Rev.Lett. 86, 5647 (2001).

[28] M.Colonna, M.DiToro, G.Fabbri and S.Maccarone, Phys.Rev. C57 1410 (1998).

[29] M.Di Toro et al., Progr.Part.Nucl.Phys. 42 125-136 (1999).

[30] Bao-An Li and C.M.Ko, Nucl.Phys. A618, 498 (1997).

[31] M.Colonna, M.Di Toro and A.Larionov, Phys.Lett. B428, 1 (1998).

[32] V.Baran, A.Larionov, M.Colonna and M.Di Toro, 
Nucl.Phys. A632 287 (1998).

[33] V.Baran, M. Colonna, M. DiToro and V.Greco, Phys.Rev.Lett. 86, 4492 (2001).

[34] Bao-An Li, Phys.Rev.Lett. 85, 4221 (2000).

[35] M.DiToro et al., Nucl.Phys. A681, 426c (2001).

[36] M.DiToro et al., Reaction mechanisms with radioactive beams: isospin effects on nuclear dynamics, Proc. Int.Symposium on Exotic Nuclei, Baikal 2001, World Sci. in press

[37] This is the case of a $R M F$ model in the Hartree approximation, mostly followed in all applications. For a discussion on the effects of the Fock terms see the recent work 38 and ref.s therein.

[38] V.Greco, M.Colonna, M.DiToro, G.Fabbri and F.Matera, Phys.Rev. C63, 035202 (2001) and Phys.Rev. C64, 045203 (2001).

[39] This choice corresponds to the $\delta$ parameters $\left(g_{\delta}, m_{\delta}\right)$ of the Bonn $C$ potential 40. We note that such value is also in quite good agreement with the density dependent study of 15 derived from a microscopic DiracBrueckner-Hartree-Fock calculation in asymmetric matter (the plateau in Fig. 2 of that ref.). In any case we are interested in the new qualitative features of the $\delta$ coupling.

[40] R.Machleidt, Adv.Nucl.Phys. 19, 189 (1989).

[41] S.Fantoni, A.Sarsa and K.E.Schmidt, Phys.Rev.Lett. 87, 181101 (2001).

[42] M.Lopez-Quelle, S.Marcos, R.Niembro, A.Bouyssy and N.Van Giai, Nucl.Phys. A483, 479 (1988).

[43] Bao-An Li, Nucl.Phys. A681, 434c (2001).

[44] Bao-An Li, C.M.Ko and W.Bauer, Jou.Mod.Phys. E7, 147 (1998).

[45] We note that in the non-relativistic case the kinetic contribution to $K_{\text {sym }}$ is actually negative, since it has the form $-\pi^{2} \rho_{0} /\left(M k_{F}\right)$ which is of the order $\sim-30 \mathrm{MeV}$. In the relativistic case it becomes slightly positive due to the density dependence of the nucleon effective mass.

[46] The solid lines in Fig.4 are not fully symmetric with respect to the $\alpha=0$ dashed line. This is due to the fact that, for fixed baryon density, the total scalar density which gives the $\sigma$ field contribution (first part of the Eq.(6)) is also slightly $N / Z$ dependent.

[47] W.Zuo, I.Bombaci and U.Lombardo, Phys.Rev. C60, 24605 (1999).

[48] E.Chabanat, P.Bonche, P.Haensel, J.Meyer, R.Schaeffer, Nucl.Phys. A627, 710 (1997).

[49] H.Huber, F.Weber and M.K.Weigel, Phys.Rev. C57, 3484 (1997).

[50] G.Sauer, H.Chandra and U.Mosel, Nucl.Phys. A264, 221 (1976).

[51] G.F.Bertsch and P.J.Siemens, Phys.Lett. 126B, 9 (1983).

[52] H.R.Jaqaman, A.Z.Mekjian and L.Zamick, Phys.Rev. C29, 2067 (1984).

[53] Of course we can have other asymmetric $N M$ instability regions, at higher baryon densities, with a different structure of the unstable modes [33. Here some interactions are even predicting quite exciting isovectorlike (species separation) unstable oscillations. Unfortunately all that appears out of the present experimental possibilities.

[54] L.D. Landau and E.M. Lifshitz, Statistical Physics, Perg- amon Press, pag. 208.

[55] G. Baym and C. Pethick, Landau Fermi-liquid theory, John Wiley and Sons, New York, 1991

[56] L.Scalone, M.Colonna and M.DiToro, Phys.Lett. B461, 9 (1999).

[57] V.Greco, Ph.D.Thesis, University of Catania 2001. 